\title{
DE LOS CAMINOS PARA LA ENSEÑANZA DE LA LÓGICA. LAS CONDICIONES DE INVISIBILIDAD DE LA LÓGICA DESDE LA ACADEMIA.
}

\author{
Dra. Diana Lizbeth Ruiz Rincón \\ dianaruizrincon@gmail.com \\ UNIVERSIDAD AUTÓNOMA DE CHIAPAS
}

Palabras clave: Didáctica de la lógica, proyectos de lógica, lógica en la academia.

Las condiciones de invisibilidad que suelen tener los contenidos filosóficos tanto en el Nivel Medio Superior como en el Nivel Superior hacen a veces ardua la labor del profesional de la filosofía en relación a su práctica docente. En este sentido, la lógica no se exime de las privaciones de relevancia como herramienta del conocer. Derivado de lo anterior, la novel Línea de Generación y Aplicación del Conocimiento "Epistemología, Lógica y Filosofía Aplicada" de la Facultad de Humanidades de la Universidad Autónoma de Chiapas, integrada por docentes de la Licenciatura en Filosofía ha generado un par de proyectos durante el 2017 denominados: Fortalecimiento de la enseñanza de la lógica en la Licenciatura en Filosofía y, Desarrollo de Materiales Didácticos para la Enseñanza de la Lógica en la Licenciatura en Filosofía, respectivamente.

La generación de los proyectos se da en condiciones de no accesibilidad a gestiones de financiamiento, cuyos productos buscan promover tanto la formación en lógica como su divulgación. Los andares y rutas para el fortalecimiento de una de las principales herramientas del filósofo, del profesional de la filosofía y del ser humano en general, donde el discernimiento de argumentos correctos o falaces, su 


\section{ARTÍCULOS}

análisis y los métodos para la demostración de la validez, juegan un papel fundamental en la configuración de expresiones del pensamiento.

La relevancia de estos proyectos de intervención, se configuran como un plan emergente en donde, en contextos similares, pueden recuperarse dichas experiencias en aras de subsanar las ausencias en la construcción de un pensamiento lógico-matemático. Las condiciones institucionales si bien no otorgan desde la formalidad un reconocimiento que visibilice la importancia de la lógica, poseen marcos ambiguos desde los cuales pueden operativizarse aquellos recursos humanos que desde la academia tienen injerencia, tal como los proyectos que la Línea de Generación y Aplicación del Conocimiento denominada: Epistemología, Lógica, y Filosofía Aplicada, ha desarrollado a partir de enero del presente año, en los que se encuentran: Fortalecimiento de la enseñanza de la Lógica en la Licenciatura en Filosofía, y el Desarrollo de Materiales Didácticos para la enseñanza de la Lógica en la Licenciatura en Filosofía.

\section{OF THE WAYS FOR THE TEACHING OF LOGIC. THE CONDITIONS OF INVISIBILITY OF LOGIC FROM THE ACADEMY.}

Keywords: Didactics of logic, projects of logic, logic in the academy.

The conditions of invisibility that usually have the philosophical content in both the Higher Level and Higher Level make the work of the philosophy professional 
in relation to their teaching practice. In this sense, logic is not exempt from privations of relevance as a tool of knowledge. Derived from the above, the novel Line of Generation and Application of Knowledge "Epistemology, Logic and Applied Philosophy" of the Faculty of Humanities of the Autonomous University of Chiapas, integrated by teachers of the Degree in Philosophy has generated a couple of projects during the 2017 denominated: Strengthening of the teaching of the logic in the Degree in Philosophy and, Development of Didactic Materials for the Teaching of the Logic in the Degree in Philosophy, respectively.

The generation of the projects is given in conditions of non-accessibility to financing, whose products seek to promote both training in logic and its dissemination. The stages and routes for the strengthening of one of the philosopher's main tools, of the professional of philosophy and of the human being in general, where the discernment of correct or false arguments, their analysis and the methods for the demonstration of validity, play A fundamental role in the configuration of expressions of thought.

The relevance of these intervention projects are configured as an emergent plan where, in similar contexts, these experiences can be recovered in order to correct the absences in the construction of a logical-mathematical thinking. The institutional conditions, although they do not grant from the formality a recognition that makes visible the importance of the logic, they have ambiguous frameworks from which they can be operative the human resources that from the academy have interference, like the projects that the Line of Generation and Application of the Knowledge denominated: Epistemology, Logic and Applied Philosophy, has developed since January of this year, in which are: Strengthening 
of the teaching of Logic in the Degree in Philosophy, and Development of Didactic Materials for the teaching of The Logic in the Degree in Philosophy.

\section{Introducción}

El siguiente documento busca exponer las Rutas para el fortalecimiento de la enseñanza de la lógica, en el que buscan superarse las condiciones de invisibilidad de la lógica desde la academia, teniendo en cuenta que se parte de los marcos de una institución formal de Educación Superior como la Universidad Autónoma de Chiapas, y que se refrenda que no será sino a través de las trayectorias académicas de los profesores-investigadores, y de la comunidad estudiantil, que podrán diseñarse y operativizarse vías de transformación para la reivindicación de contenidos cuya primacía ha sido relegada por contenidos de "moda" o mayormente "populares". Para ello, el presente documento se organiza en tres apartados principales.

El primero de ellos: Del institucionalismo de la institución a la institucionalidad, abordará los aspectos institucionales del programa del cual emergen las rutas para el fortalecimiento de la enseñanza de la lógica, tomándola como un campo fundamental dentro de la filosofía, e indispensable para la formación básica de cualquier estudioso de esta disciplina. En segundo lugar, se establecen las condiciones con las que se ha creado la Línea de Generación y Aplicación del Conocimiento nombrada: Epistemología, Lógica y Filosofía Aplicada. Cerrando así con la presentación de los dos proyectos que se han registrado por parte de ésta novel LGAC. 


\section{Del institucionalismo de la institución a la institucionalidad} La Universidad Autónoma de Chiapas, mejor conocida como la UNACH es una de las Instituciones de Educación Superior (IES) con mayor prestigio y renombre en el Estado. Es también un referente necesario e indispensable para el análisis de la situación de la Educación Superior en Chiapas, ya que su historia y trayectoria académica marcan un hito en lo que a la formación de profesionistas en diversas ramas del conocimiento se refiere; así como a la producción y divulgación del conocimiento.

Creada en 1975, el Dr. Manuel Velasco Suárez, Gobernador constitucional del estado de Chiapas, la UNACH fue inaugurada en abril del mismo año, realizándose con ello las primeras acciones entre las que incluían la construcción del inmueble y adaptación de edificios, así como el nombramiento de funcionarios universitarios. A más de 40 años de distancia ésta máxima casa de estudios se ha consolidado como una de las IES públicas más representativas de la región.

Actualmente, dentro de la estructura institucional se encuentra la Dirección General de Investigación y Posgrado (DGIP), cuyos objetivos se centran en promover e impulsar la investigación y la profesionalización, principalmente mediante la oferta de posgrados y el registro y control de proyectos de investigación. De esta manera, al interior de la estructura organizacional institucional, cada Facultad cuenta con una Coordinación de Investigación y Posgrado encargada, entre otras actividades, de reportar los registros y seguimiento de proyectos de investigación y sus resultados ante la DGIP. 
Para ello debe procederse con el registro del proyecto de investigación, a través de los formatos DI-F1-RE ${ }^{1}$ o DI-F1-RP ${ }^{2}$, cuya distinción radica en que para el primer caso se refiere a proyectos de investigación financiados con recursos externos, mientras que el segundo habrán de desarrollarse y ejecutarse con recursos propios del investigador o grupo de investigadores involucrados. Junto al registro del proyecto debe entregarse el formato DI-F2, que será el protocolo en el que deberán detallarse los elementos teórico-metodológicos del proyecto, sus objetivos y metas, así como su calendarización, entre otros.

De las condiciones institucionales para la investigación, los programas de pregrado ofertados por las distintas escuelas y facultades configuran un escenario idóneo desde el cual los profesores-investigadores habrán de articular los distintos objetos de estudio con relación a los marcos teóricos-metodológicos y conceptuales propios de sus campos disciplinares. Para el caso de la Facultad de Humanidades, Campus VI, cuya oferta académica se integra por siete licenciaturas (seis programas presenciales y uno a distancia) ${ }^{3}$, una especialidad, una maestría y un doctorado ${ }^{4}$, la Licenciatura en Filosofía integrará una de las siete primeras. De esta manera, ha sido posible situar el contexto institucional (al menos de manera somera) en el que el programa que nos ocupa tiene lugar.

\footnotetext{
${ }^{1}$ DI: Dirección de Investigación; F1: Formato 1; y, RE: Recurso Externo.

2 DI: Dirección de Investigación; F1: Formato 1; y, RE: Recurso Propio.

3 Siendo de modalidad presencial: Licenciatura en Pedagogía, Licenciatura en Comunicación, Licenciatura en Bibliotecología y Gestión de Información, Licenciatura en Lengua y Literatura Hispanoamericana, y la Licenciatura en Filosofía. Y de modalidad a distancia: Licenciatura en Tecnologías de Información y Comunicación Aplicadas a la Educación.

${ }_{4}^{4}$ Todos ellos inscritos en el Padrón Nacional de Programas de Calidad del Consejo Nacional de Ciencia y Tecnología (PNPC-CONACyT).
} 
Aunado a lo anterior, nos encontramos en condiciones de abordar aquellos aspectos institucionales que marcan al curriculum formal, donde desde la trayectoria del profesor-investigador en el ejercicio de docente pueden tener lugar prácticas para la construcción de rutas de transformación. Otro modo de referir lo anterior sería a partir del modo en que ese "reduccionismo" es normado e instituido a partir del diseño e implementación de Planes y Programas de Estudio, y del cómo desde la práctica docente pueden generarse adecuaciones en el currículum oculto mediante el ejercicio de las trayectorias académicas en los parámetros de la institución.

De esta manera, sumado a que la Licenciatura en Filosofía inicia su primera generación en agosto de 2011, contando a la fecha con cuatro generaciones de egresados, el Plan de Estudios no ha sufrido más que una actualización en 2012 que obedece principalmente a un requerimiento estructural más que de contenido; es decir, su actualización operó en relación a la reorganización de las asignaturas que integran la malla curricular, y no a la modificación, incremento o reelaboración de las mismas. Lo anterior permite identificar una sola asignatura de lógica que se imparte en primer semestre y que por nombre lleva: lógicas, suponiendo con ello el abordaje de contenidos de introducción a la lógica, lógicas clásicas y lógicas no clásicas (en su división más general) e inclusive filosofía de la lógica en aproximadamente 36 sesiones de dos horas cada una. Lo cual supera por mucho las aspiraciones no solo metodológicas o instrumentales de su aproximación, sino también los aspectos teóricos y conceptuales que lo fundamentan.

Por lo anterior, la presente exposición hace énfasis en que no todo está perdido, que puede ser incluso desde las restricciones institucionales dadas por la 
normatividad inherente a los procesos formativos, donde desde el ejercicio docente pueden promoverse actividades y proyectos complementarios que coadyuven al desarrollo de habilidades lógico-matemáticas en los estudiantes, así como la procuración y generación de condiciones extracurriculares que permitan ampliar la extensión temporal y de contenido en lo que a la enseñanza de la lógica se refiere, entendiéndola como una herramienta fundamental para el quehacer del filósofo y del profesional de la filosofía. De esta manera ha sido posible ir justificando las razones y configurando la Línea de Generación y Aplicación del Conocimiento (LGAC) denominada: Epistemología, Lógica y Filosofía Aplicada. LGAC que cabe mencionar, no figura en el Plan de Estudios de la Licenciatura en Filosofía, pero que, al registrar un proyecto de investigación ante la DGIP cuyos objetivos y productos estén centrados en algún objeto de estudio que no se encuentre explicitado en el instrumento institucional, su ausencia marcará parámetros de permisibilidad en los que los productos de las investigaciones encuadradas en dicha LGAC serán las bases para su futura consolidación.

Por ello, hablar "del institucionalismo de la institución, a la institucionalidad" es un modo de referirnos al énfasis dado a la práctica docente, en tanto ejecución del roll de profesor-investigador, cuya operatividad dentro de los marcos institucionales permite la actuación dentro de los lineamientos de las LGAC del Plan de Estudios, e incluso la generación (fortalecimiento y consolidación) de LGAC noveles como rutas de transformación desde la investigación hasta su implementación, siendo este último aspecto el que habilita la construcción de trayectorias académicas en los procesos formativos cuyos vacíos curriculares pueden ser atendidos desde la misma práctica investigativa. 


\section{La novel Línea de Generación y Aplicación del Conocimiento "Epistemología, Lógica y Filosofía Aplicada"}

Como una estrategia que desde las ambigüedades de la norma puede instaurarse a partir de la producción, como la atención a un vacío en el currículum respecto a la enseñanza e la lógica, ha sido la creación de la LGAC: Epistemología, Lógica y Filosofía Aplicada. En el que uno de sus objetos de estudio se centra en la Lógica, a manera de visibilizar no sólo este campo de la filosofía, sino de reivindicar su relevancia dentro de los marcos institucionales de formación básica el filósofo.

Es importante referir el contexto desde el cual el Plan de Estudios marca las líneas que cultivará la Licenciatura en Filosofía, a saber: Filosófica-antropológica, Epistemológica-ética, Ética-ecológica, Hermenéutica-filosófica, Problemática sobre la crisis del cuidado de sí, Filosofía política, Filosofía de la ciencia, Filosofía de la educación, Filosofía tecnológica y Didáctica de la filosofía. (p.43) Teniendo pues presente la omisión de una LGAC que cultive la investigación y aplicación de la lógica, de lo anterior se sigue pues el señalamiento de la no visibilidad de la lógica dentro del curriculum formal de este programa académico.

Tenemos pues, por un lado, la escaza presencia de la enseñanza de la lógica en el PE, apreciable en la malla curricular, que ya en el espacio anterior había sido referido, marcando que:

En la malla curricular del vigente plan de estudios de la Licenciatura en Filosofía (2012) de la Universidad Autónoma de Chiapas (UNACH), se contemplan dos asignaturas que aluden a la Lógica: "Lógicas", y "Lógica informática". La asignatura "Lógicas" forma parte de la "Formación Básica 
Común" y se encuentra en el primer semestre. Por otra parte, la asignatura "Lógica informática" se encuentra en el sexto semestre de la "Opción de Formación Profesional B: Logotutoría", específicamente en el "Área de Formación Filosofía del Lenguaje y la Comunicación". Es importante notar la distancia entre una asignatura y otra, mientras que una está en el primer semestre, la que le sigue está en el sexto semestre. Esto representa un doble problema: el primero, no hay la debida continuidad de los conocimientos aprendidos. Para cuando los estudiantes vuelvan a trabajar lógica, ya habrán olvidado lo aprendido en el primer semestre. El segundo problema, es que sólo los estudiantes que eligen la línea de "Filosofía Aplicada" pueden llevar la siguiente asignatura de lógica, por lo que los demás estudiantes que eligieron las dos líneas restantes, desconocen los contenidos que son estrictamente necesarios para su formación como filósofos. (Ruiz Rincón, Cañas Muñoz, Ruiz Sosa, y Canela Morales, 2016, p. 211)

De este modo, y continuando con la idea anterior, hemos identificando aquellas líneas que desde lo instituido prevén las condiciones institucionales que procuraran su desarrollo, encontrando en ellas a partir de la síntesis temática y de contenido, la relevancia de los campos de las filosofías aplicadas y la epistemología, por lo que ésta LGAC habrá de buscar pues, por un lado, atender los objetos de estudio propias de la epistemología y la denominada filosofía aplicada (o filosofías aplicadas), teniendo como elemento central y visible a la lógica.

En este sentido, incentivar, nutrir y fortalecer la enseñanza de la lógica a través de la generación de proyectos en los que más que una práctica encerrada en sí misma, se constituya en una actividad constante cuyos productos permitan a los egresados llevar a otros espacios, principalmente al nivel medio superior, actividades o programas de formación en esta disciplina. Cabe mencionar en este 
contexto que, si bien la lógica no se circunscribe o reduce a la lógica proposicional, en su aspecto meramente formal, existen muchas y diversas aristas en el estudio e investigación de la lógica que son relevantes, como: teoría de la argumentación, lógica clásica y lógicas no clásicas, filosofía de la lógica, historia de la lógica, lógica formal, lógica no formal, e inclusive lógica informal, entre otros.

Recapitulando, al registrar algún proyecto de investigación ante la DGIP, existe en el formato de registro (DI-F1-RP), un apartado en el que debe marcarse el título del proyecto, la vigencia (fecha de inicio y de conclusión, sugiriéndose no más de 12 meses de extensión), el área del conocimiento en la que se inscribe, el tipo de investigación, el cuerpo académico al que pertenece el Responsable Técnico $^{5}$; siendo posible, en el caso de no contar con un CA (como en este programa académico) que el docente que forme parte de la plantilla docente del ciclo escolar en el que se registra el proyecto, pueda registrarse únicamente la LGAC que se estará cultivando, permitiéndose con ello el registro de los trabajos iniciales de una línea de investigación que a la postre habrá de permitir conformar un grupo de investigación.

Dicha flexibilidad permite que programas académicos de reciente creación inicien proyectos de investigación que habrán de labrar líneas que a su vez fortalecerán los procesos formativos de la comunidad estudiantil. De ahí que haya sido posible no solo el registro de una emergente LGAC en la Licenciatura en Filosofía de la UNACH, sino también los trabajos de investigación desarrollados por los docentes temporales con perfil de investigadores, y que han focalizado los

\footnotetext{
${ }^{5}$ Que será el docente responsable de rendir los informes de avances y conclusión del proyecto.
} 
primeros proyectos registrados al tratamiento de una de las disciplinas más endeblemente tratadas por el currículo, la lógica.

El primero de los proyectos parte pues de un diagnóstico sobre el aprendizaje de la lógica desde el cual se justifica la elaboración del Breve Manual de Lógica Matemática (Ruiz Rincón, Canela Morales, y Cañas Muñoz, 2017), cuyo impacto busca situarse en el fortalecimiento de los procesos formativos de los estudiantes respecto a los contenidos de la lógica en un contexto de invisibilización. El segundo proyecto está constituido por la investigación para la elaboración de cuadernillos de trabajo que permitirán atender de manera más puntual cada uno de los apartados que integran los temas que aborda el Breve Manual de Lógica Matemática (Ruiz Rincón, Canela Morales, y Cañas Muñoz, 2017).

Así, con su reciente aparición, la LGAC denominada: “Epistemología, Lógica y Filosofía Aplicada", es un modo en el que desde la generación de trayectorias académicas, como rutas para el fortalecimiento de la enseñanza y la investigación de dichos campos de la filosofía, ha sido viable la reivindicación de la lógica en la agenda de la práctica docente.

\section{Los proyectos 2017, una ruta para la visibilidad}

Desde los inicios del 2017 se tomaron acuerdos y acciones por parte de la plantilla docente que integra la Licenciatura en Filosofía de la Universidad Autónoma de Chiapas, con el objeto de atender las vicisitudes a las que se ha venido enfrentando éste curriculum, a partir de las configuraciones de las distintas trayectorias 
académicas en las que las rutas para la visibilidad habrán de operar a modo de construcción, generación y gestión de proyectos académicos y de investigación.

Para finales del año 2016 se realizaron una serie de reuniones de trabajo intenso que derivó en la elaboración del primer proyecto intitulado: Fortalecimiento de la enseñanza de la Lógica en la Licenciatura en Filosofía, registrado con una vigencia que daba inicio el nueve de enero de 2017 y concluía el siete de julio del mismo año, se acordó a partir de ese momento iniciar los trabajos de la LGAC: Epistemología, Lógica y Filosofía Aplicada estableciendo para ello la meta de registrar dos proyectos por año, permitiendo con ello mantener por un lado el interés en dichas actividades investigativas sobre la temática propuesta por docentes que, de modo rotativo, es decir, desde sus trayectorias, habrán de establecer las rutas de acción y el proyecto a desarrollar.

Inscrita en el área del conocimiento número "cero, cuatro": Humanidades, Educación y Ciencias de la Conducta; éste primer proyecto se considera como una investigación de tipo básica, cuya adscripción a un Cuerpo Académico (de reciente creación, en consolidación o consolidado) o Grupo de Investigación es inexistente por ser un programa académico de reciente creación y sin las condiciones institucionales y laborales requeridas para la conformación de éstos.

Aunado a lo anterior, cabe recalcar que las mismas condiciones institucionales, aunque restrictivas, también poseen un sesgo de posibilidad de ruptura y generación de rutas propias que han sido apropiadas por este grupo docente para generar marcos de visibilidad de y en torno a la enseñanza de la lógica en el contexto universitario formal ya referido. 
De esta manera, el objetivo general del primer proyecto se plantea en términos e desarrollar y fortalecer la enseñanza de la Lógica en la Licenciatura en Filosofía de la Universidad Autónoma de Chiapas (UNACH). Lo anterior, a través del diagnóstico temprano, la intervención y análisis crítico de la visión incompleta que de la lógica se tiene en el actual plan de estudios. En ese sentido, el objetivo general consistió en presentar un plan emergente para la formación de filósofos en un contexto más amplio como lo es el de la lógica en sus sentidos conceptual y procedimental. Dicho plan hubo de consistir en la impartición de talleres extracurriculares, desarrollo de asignaturas que estructuren e interpreten sistemas lógicos y talleres extraordinarios con profesionales del campo de la lógica.

La justificación a lo anterior se centra en la ausencia de un tratamiento de la lógica (formal, informal y argumentativa) dentro del plan de estudios vigentes. Lo anterior, sumado al escaso compromiso en el programa de lógica que reciben los alumnos durante su paso por el nivel medio superior, permite anticipar una condición de rezago, respecto del dominio de la lógica, en la que los estudiantes de nuevo ingreso a la Licenciatura en Filosofía habrán de arribar. Estos son, pues, los principales indicadores de la situación que enfrenta la Licenciatura en Filosofía, UNACH. El objetivo a largo plazo a cumplir con este programa emergente es la apropiación de una tradición filosófica en lógica que promueva en los egresados de esta licenciatura no sólo movilidad estudiantil, sino también un mayor campo de inserción laboral.

En un sentido metodológico, los objetivos específicos trazados permitirán exponer tres momentos centrales que integraron dicho proyecto: en primer lugar, se optó por diagnosticar a partir del establecimiento de la variable relacional en la 
que se determine el nivel de retención de los contenidos de la asignatura de lógica cursada en primer semestre, respecto a ubicación del semestre en que se encuentren los alumnos evaluados. En segundo lugar, se aplicaron los resultados del diagnóstico para elaborar un manual que permita recuperar y reforzar los contenidos de lógica que todo estudiante de la Licenciatura en Filosofía debe dominar; buscando con ello intervenir con estudiantes y docentes en el fortalecimiento de la lógica a través de la impartición de un taller semestral para el caso de los primeros, y un taller inter-semestral para el caso de los segundos.

Así, derivado del análisis, se determinaron una serie de metas con las que llevar a cabo acciones que permitan compensar las deficiencias en los procesos de enseñanza-aprendizaje de la lógica como instrumento de análisis de argumentos y el desarrollo de habilidades del razonamiento. Para ello fue necesario llevar a cabo un diagnóstico, a partir de la aplicación de una evaluación diagnóstica a los estudiantes que se encuentren entre el segundo y el séptimo semestre de la Licenciatura en Filosofía.

La enseñanza y el estudio de la lógica en la formación del filósofo habrán de tocar también los aspectos relacionados al modo en que generan y expresan sus propios procesos de pensamiento, reflexionando, argumentando y generando producciones principalmente escritas, teniendo como segunda meta la publicación de un Breve Manual de Lógica Matemática, que permita coadyuvar en los procesos formativos tanto de estudiantes como de los docentes. La tercera meta consistirá en la impartición de dos talleres, uno para docentes y otro para estudiantes, lo que permitirá la implementación de acciones paliativas que permitirán agotar los aspectos introductorios de la enseñanza de la lógica. 


\section{ARTÍCULOS}

Las anteriores actividades se desarrollaron, como se había expuesto líneas arriba, de enero a junio del año 2017, cumplimentándose en tiempo y forma cada una de las actividades, en las que se incluyeron: Aplicación de examen diagnóstico, Diagnóstico de conocimientos de lógica en estudiantes de segundo semestre en adelante, Registro de talleres, Publicación de Manual, Impartición de Taller 1, Impartición de Taller 2, Informe técnico e Informe Final. Resultando imperativo señalar la integración y participación de un estudiante con la elaboración de su reporte final de investigación y la defensa de tesis correspondiente en tiempos y formas establecidos. Finalmente, el primer proyecto concluyó a finales de junio, integrándose la evidencia y entregándose el informe final por parte de la Responsable Técnico del Proyecto, la Dra. Diana Lizbeth Ruiz Rincón.

Posteriormente se procedió con el registro del segundo proyecto de investigación de la LGAC: Epistemología, Lógica y Filosofía Aplicada, a denominarse: Desarrollo de Materiales Didácticos para la enseñanza de la Lógica en la Licenciatura en Filosofía. Cabe mencionar en este contexto que, derivado de la elaboración del Breve Manual de Lógica Matemática (Ruiz Rincón, Canela Morales, y Cañas Muñoz, 2017), se determinó la necesidad de desarrollar una serie de cuadernillos que fungieran como auxiliares didácticos en el abordaje de cada uno de los capítulos que integran el manual señalado.

De este modo, y con el Mtro. Manuel Arnulfo Cañas Muñoz fungiendo como responsable técnico, con este proyecto de investigación en términos generales pretende dar continuidad a los productos entregados a partir de otros proyectos dirigidos a atender las necesidades formativas en tópicos lógicos, desde la Licenciatura en Filosofía de la Universidad Autónoma de Chiapas (UNACH). 
En especial el Breve Manual de Lógica Matemática (Ruiz, Cañas, Canela, 2017), y el "Taller de Lógica Avanzada", tienen una continuación directa en este proyecto.

La importancia de la Lógica como metodología del análisis filosófico, se puede comparar con el papel que desempeñan las matemáticas en las Ciencias Naturales. Es ese carácter metodológico el que implica acompañar los rubros y temas abordados en el Manual mencionado con ejercicios y ejemplos que permitan al estudiante o al interesado en la Lógica, desarrollar pericia a partir de la práctica. Esos ejercicios serán tomados a partir de nuestra propia práctica docente, y también a partir de las experiencias obtenidas en el "Taller de Lógica Avanzada". Los ejercicios se agruparán en una serie de cinco cuadernillos.

Todos estos elementos permitirán no sólo suplir algunas de las carencias formativas en lógica de la Licenciatura en Filosofía de la $\mathrm{UNACH}$, sino que también permitirán consolidar la práctica y una posible tradición en el estudio de la Lógica Matemática desde la propia UNACH. Es por ello que este proyecto de investigación también toma en cuenta la participación de algunos de nuestros integrantes en los eventos organizados por la Academia Mexicana de la Lógica (AML).

Teniendo en cuenta la metodología del presente proyecto que opera como una extensión del proyecto anterior, parte de una serie de investigaciones y documentaciones que servirán para elaborar cinco cuadernillos publicables en el siguiente orden: 1) cuadernillo de lógica aristotélica; 2) cuadernillo de tablas de verdad; 3) cuadernillo de lógica proposicional; 4) cuadernillo de lógica cuantificacional; y 5) cuadernillo de estrategias didácticas. Todos estos cuadernillos que se publicarán por autor guardan una estrecha vinculación con el Breve manual 
de lógica matemática; puesto que son una secuencia del mismo, en el sentido de que el libro aporta la mirada teórica y los cuadernillos están constituidos por ejercicios que procuran ser una aplicación de lo teorizado en el libro. Cabe señalar que este libro fue publicado como producto del proyecto anterior.

Seguido de esto, se presentará mediante una ponencia el libro aludido en el Congreso Internacional de Lógica convocado por la Academia Mexicana de Lógica (AML) que será celebrada en la Universidad Popular Autónoma de Puebla (UPAEP) en noviembre de 2017. Además, se buscarán otros espacios para su presentación a la comunidad académica.

Finalmente, el proyecto culminará con dos talleres de lógica, uno destinado a los estudiantes y el otro, a los docentes de la universidad e invitados del Nivel Medio Superior. Mientras que el primer taller se centrará en la lógica básica; el segundo, operará en un sentido didáctico. Con esto, se busca poner en marcha la operatividad del producto obtenido en el primer proyecto de lógica para coadyuvar en la formación de los estudiantes y la actualización docente.

Como proyecto de continuación, el fortalecimiento de la enseñanza de la Lógica en la Licenciatura en Filosofía de esta máxima Casa de Estudios ha marcado como metas la generación de una serie de 5 cuadernillos de trabajo tocando los temas: lógica aristotélica, tablas de verdad, lógica proposicional, lógica cuantificacional y estrategias didácticas; lo anterior con el fin de coadyuvar a los estudiantes, con ejercicios prácticos y a docentes con la presentación de estrategias didácticas para la enseñanza de la lógica, para el desarrollo y ejecución del "Breve manual de lógica matemática". 


\section{ARTÍCULOS}

Seguidamente se buscarán espacios académicos para la presentación y difusión del "Breve manual de lógica matemática", así como para compartir experiencias formativas y medios de superación de las condiciones de la formación lógica en el pre-grado. Aunado a lo anterior, se promoverá la impartición de dos talleres, el primero que, al ser promovido para los estudiantes con contenido de lógica básica, utilizará como material de trabajo el "Breve manual de lógica matemática". Mientras que, para el segundo taller de didáctica de la lógica para docentes, se abordaran distintos enfoques didáctico-metodológicos para el tratamiento y enseñanza de la lógica mediante el trabajo colegiado y el intercambio de experiencias.

Por ser un proyecto que a la fecha en la que se redacta el presente documento se encuentra en proceso de ejecución, puede únicamente referirse con fines informativos el periodo de vigencia, que comprende del primero de mayo al quince de diciembre del año 2017, sobreentendiéndose con ello su "en realización". En este sentido pueden considerarse abordadas dos rutas de visibilidad de las condiciones que guarda la lógica en un contexto determinado, cuyas posibilidades se abren desde el actuar mismos de las trayectorias académicas que identifican al campo de la lógica como un objeto de estudio vigente, tanto en los marcos teóricos, metodológicos, como prácticos y de aplicación.

\section{Conclusiones}

Hablar respecto a las rutas para el fortalecimiento de la enseñanza de la lógica, como aquellas condiciones de invisibilidad de la lógica desde la academia, es un modo de hacer referencia a las restricciones a las que la lógica, la filosofía o las 
humanidades en general se encuentran en los ámbitos institucionales de la educación formal, en donde los discursos hegemónicos e ideologías imperantes marcan las prioridades de las políticas educativas a un nivel macro como micro.

Sin embargo, el presente documento busca ser el referente de un esfuerzo que desde la misma institucionalización de las prácticas formativas en filosofía, particularmente de la lógica, ha encontrado vías alternas de realización que ejecuta desde la misma práctica docente, en donde, al hacer agencia se reconfigura el curriculum y se desarrollan trayectorias académicas que harán las veces de portavoces para la atención de aquellos contenidos que no son "priorizados" o integrados en los esquemas de formación en el campo educativo.

De la restricción de la institución formal hacia la agencia, implica en sí mismo un esfuerzo por parte de los docentes interesados en cultivar algún campo del conocimiento que se encuentre en condiciones de invisibilidad, construyendo o generando las condiciones internas para la configuración de líneas de generación y aplicación del conocimiento que buscarán ser cultivadas no ya exclusivamente por el profesor-investigador, sino por aquellos estudiantes inclinados por la temática y que puedan en ellas construir un proyecto de investigación y por qué no, de profesión.

Así, los proyectos antes referidos son un esfuerzo por parte de la plantilla docente que presta sus servicios profesionales en la Licenciatura en Filosofía de la Facultad de Humanidades, Campus VI, de la Universidad Autónoma de Chiapas, y en cuyos elementos integradores se han adherido los intereses personales de investigación, permitiéndose con ello generar las condiciones internas para la 
| Cuatrimestral Facultad de Humanidades Campus VI Reserva de Derechos al Uso Exclusivo No.: 04-2018-060814012200-203| ISSN: 2594-1755

Año II | Número 4 | Septiembre-Diciembre 2018

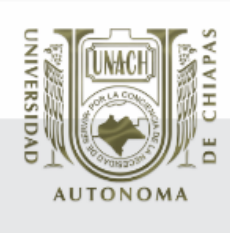

\section{ARTÍCULOS}

continuidad y seguimiento de los proyectos e intereses investigativos que la LGAC habrá de cultivar.

\section{Referencias}

Cabanzo Vargas, A. (2009, julio-diciembre), “La enseñanza de la lógica y el análisis del texto argumentativo". En Revistas Actualidades Pedagógicas. No. 54, pp.159-172. Disponible en línea en

http://revistas.lasalle.edu.co/index.php/ap/article/view/959/866

CEFTA (2016), Plan de estudios. Disponible en línea en http://filosofiacefta.com/index/index.php/oferta-academica/licenciaturafilosofia/plan-de-estudios

Château, J. (2005), Los grandes pedagogos. Trad.: Ernestina de Champourcín. México: FCE.

Copi, I. (1996), Lógica Simbólica. México: Compañía Editorial Continental, S.A. de C.V.

Copi, I., \& Cohen, C. (2013), Introducción a la Lógica. México: Limusa.

Delval, J. (2004), Los fines de la educación. México: Siglo XXI.

Kant, I. (2013), Pedagogía. Trad.: Lorenzo Luzuriaga y José Luis Pascual. Madrid: Akal.

Raymundo Morado, "La lógica en México: Raíces, logros y posibilidades" en http://www.filosoficas.unam.mx/ morado/Papers/RaicesLogros.htm

Rayo, A. (2004), "Formalización y lenguaje ordinario". En Orayen, R. \& Moretti, A. (ed.). Filosofía de la lógica. Madrid: Trotta.

Ruiz Rincón, D. L. (2011), Diagnóstico de la situación de la enseñanza de las asignaturas de corte filosófico: Ética y valores I y II, en el Colegio de Bachilleres de Chiapas, planteles 01 Terán y 13 Oriente. [Tesis de maestría]. Universidad Autónoma de Chiapas. 
